\title{
The Calculus of Handshake Configurations
}

\author{
Luca Fossati ${ }^{1,2, \star}$ and Daniele Varacca ${ }^{2}$ \\ 1 Dip. di Informatica - Università di Torino, Italia \\ fossati@di.unito.it \\ 2 PPS - CNRS \& Univ. Paris Diderot, France
}

\begin{abstract}
Handshake protocols are asynchronous protocols that enforce several properties such as absence of transmission interference and insensitivity from delays of propagation on wires. We propose a concurrent process calculus for handshake protocols. This calculus uses two mechanisms of synchronization: rendez-vous communication à la $\mathrm{CCS}$, and shared resource usage. To enforce the handshake discipline, the calculus is endowed with a typing system.

We provide an LTS semantics of the calculus and show that typed processes denote handshake protocols. We give the calculus another semantics in terms of a special kind of Petri nets called handshake Petri nets. We show that this semantics is complete and fully abstract with respect to weak bisimilarity.
\end{abstract}

Keywords: Handshake protocols, Petri nets, process calculus, types.

\section{Introduction}

Asynchronous circuits are used to design systems where the local activity of each subunit is not restrained by some global condition, like the long time intervals imposed by a system clock. When designing such systems, one has to face several questions. How do we know when a message we sent has reached its destination so that we can use the same channel again, i.e. how can we avoid transmission interference? How can we ensure the correct behavior regardless of computational speeds of single modules and propagation delays over wires, i.e. how can we enforce delay-insensitivity?

Handshake protocols try to answer these questions by imposing an interactive communication discipline. In particular, the protocols require that after a circuit has sent a message on a channel, it has to wait for a confirmation that the message was received before sending again on the same channel. This requirement alone is enough to rule out transmission interference. For their simplicity and efficiency, handshake protocols have been employed by enterprises like Philips and Sun in the development of a series of VLSI chips [9].

The first attempts to formalize delay-insensitive protocols and their properties employed trace sets [17]. In particular, the first model to specifically address the handshake case was given in [18]. Trace models have been able to neatly formalize the properties of handshake protocols which ensure delay-insensitivity, but so far they have failed in representing correctly their composition [4].

\footnotetext{
^ Corresponding author.
} 
To overcome this limitation, we propose an alternative approach to modeling handshake protocols: we propose a process calculus inspired by Robin Milner's Calculus of Communicating Systems (CCS) [13]. Similarly to CCS, our calculus defines concurrent processes that communicate via rendez-vous channels. However, in order to ensure the handshake discipline, the calculus features another synchronization mechanism, by means of shared resources, reminiscent of coordination languages like Linda [7]. Also, the calculus is endowed with a linear typing system, inspired by [11[19]. These design choices allow to express the external behavior of a handshake protocol, along with a more complex internal behavior.

We say that this is the first independent syntactical description of the handshake behavior, as our handshake configurations are independent from any semantical interpretation while the handshake behavior is ensured by the typing system. This was not the case in previous process algebras for handshake protocols [18 10], where only processes whose trace semantics satified the handshake behavior were considered, thus processes were trace sets and inevitably suffered from the compositionality problems observed in the underlying trace model.

We then compare our calculus and the trace model by defining, for each configuration, the corresponding set of quiescent traces, i.e. the traces corresponding to computations that may not be extended with an output event. We show that this quiescent trace semantics is sound w.r.t. Van Berkel's definition [18]. By means of an example, we show that quiescent trace equivalence is not a congruence w.r.t. parallel composition. This confirms the intuition that trace models of handshake protocols are not informative enough, and their branching structure needs to be taken into account. Indeed weak bisimilarity is a congruence for our calculus.

To show the expressive power of the calculus, we give it a Petri net semantics, where the handshake discipline is imposed by restricting a net's external structure. We studied this model in details in a previous work [6], where we showed that it captures precisely the behavior of a protocol, in the sense that there is a protocol for each net and a net (possibly with an infinite number of places and/or transitions) for each protocol. In this work we show that there is a correspondence between handshake processes and finite Petri nets, in the sense that for each finite handshake Petri net, there is a weakly bisimilar process.

The graphical approach to the formal analysis of asynchrony is not a new one ([1],[12],...). In particular Dan Ghica developed a language for asynchronous hardware design by taking inspiration from the Geometry of Interaction and handshake circuits [8]. However his goal was to improve previous hardware design languages [183] and not to capture all handshake behaviors.

The paper is structured as follows. In Section 2 we recall the first formalization of handshake protocols as we introduce the notion of handshake language. In Section 3 . we present syntax, operational semantics and type system of our calculus. We show that the set of quiescent traces of a typed configuration is a handshake language. We show that weak bisimilarity is a congruence, while quiescent trace semantics is not. In Section 4, we present handshake Petri nets, with some examples to show how they work. In Section 5, we present the interpretation of the calculus into handshake nets, and we show that it is fully abstract with respect to weak bisimilarity. To conclude, we 
show the universality of the semantics, by showing that every bisimilarity class of finite handshake nets is denoted by a process.

\section{Handshake Protocols}

In this section we recall the background properties of handshake protocols and introduce the notion of handshake language.

Definition 1. A handshake structure is a pair $\langle$ Ports, $d\rangle$, where Ports is a finite set of ports and the function $d:$ Ports $\rightarrow\{!, ?\}$ determines a direction for each port, active or passive.

As we shall see, active ports are allowed to start a communication, while passive ports are initially waiting. For the rest of this section let $\langle$ Ports, $d\rangle$ be a handshake structure. For each port $a$, there are two possible messages: $a$ (input message), and $\bar{a}$ (output message). Let $t$ be a finite trace on the alphabet of messages $\cup_{a \in \text { Ports }}\{a, \bar{a}\}$. $t$ is a handshake trace on $\langle$ Ports, $d\rangle$ if for all $a \in$ Ports:

- $t \uparrow\{a, \bar{a}\}=\bar{a} a \bar{a} a \ldots$ when $d(a)=$ !;

- $t \uparrow\{a, \bar{a}\}=a \bar{a} a \bar{a} \ldots$ when $d(a)=$;

Given a set of traces $S$ we write $S \leq$ for its prefix-closure. Let $\sigma$ be a set of handshake traces, $s \in \sigma^{\leq}$is passive in $\sigma$ if and only if there is no extension of $s$ in $\sigma$ obtained by appending an output message: $\forall s \cdot m \in \sigma^{\leq}, m$ is an input message. We write $\operatorname{Pas}(\sigma)$ for the set of passive traces in $\sigma^{\leq}$.

We define $\mathbf{r}_{\text {Ports }}$ as the smallest binary relation which is closed by reflexivity, transitivity and concatenation and such that for any distinct ports $a, b \in$ Ports:

1. $a \bar{b} \mathbf{r}_{\text {Ports }} \bar{b} a$;

2. $\bar{a} \bar{b} \mathbf{r}_{\text {Ports }} \bar{b} \bar{a}$;

3. $a b \mathbf{r}_{\text {Ports }} b a$

We say that $s$ reorders $t$ in Ports if $s \mathbf{r}_{\text {Ports }} t$.

Let $s$ be a handshake trace and $a \in$ Ports. We write $a \mathbf{x}_{\text {Ports }} s$ if $s a$ is still a handshake trace. Finally:

Definition 2. A handshake language $\sigma$ on $\langle$ Ports, $d\rangle$ is a non-empty set of finite handshake traces on $\langle$ Ports, $d\rangle$ such that:

1. $\operatorname{Pas}(\sigma) \subseteq \sigma$ (closed under passive prefixes);

2. $\left(t \in \sigma \wedge s \mathbf{r}_{\text {Ports }} t\right) \Rightarrow s \in \sigma$ (reorder closed);

3. $\left(s \in \sigma^{\leq} \wedge a \mathbf{x}_{\text {Ports }} s\right) \Rightarrow s \cdot a \in \sigma^{\leq}$(receptive).

Closedness under passive prefixes, rather than under any prefix as it is usually the case for trace semantics, allows us to represent maysmust nondeterminism. We want to represent systems which are able not only to make an exclusive choice between two outputs, but also to choose between sending an output and not doing anything.

The intuition is thus that a trace in a handshake language represents a quiescent execution of a protocol, that is an execution that ends in a state in which the system may 
decide to wait for more inputs before sending any output. By definition, after a passive trace the system cannot do anything but receiving. Then all passive traces correspond to quiescent executions.

Reorder-closedness says that a message $m^{\prime}$ cannot "block" a message $m^{\prime \prime}$ on a different channel unless $m^{\prime}$ is an input and $m^{\prime \prime}$ an output. The intuition is that inputs may carry necessary information and thus may block, while the transmission of an output may require informations and can thus be blocked.

Finally, receptiveness means that whenever it is the environment's turn to send a message, the system must be ready to accept it.

Definition 2] is like VanBerkel's original definition of handshake process [18] without data-passing. No satisfactory definition of composition for handshake languages exists. In particular the definition given by VanBerkel is not associative, as shown by the first author [4] using a counter-example by Roscoe [16].

In the following sections, handshake languages will be used as a yardstick against which to measure the correctness of other descriptions of handshake protocols.

\section{The Calculus}

In this section we provide the formal definition for our Calculus of Handshake Configurations $(\mathrm{CHC})$. We stress that we do not model data-passing as we are only interested in the communication protocol. The calculus is endowed with two communication mechanisms. Besides the external communication via rendez-vous channels, there is also a form of internal communication, invisible to the outside, where actions may require resources in order to be performed and may release resources for other actions to use. This is necessary to model internal synchronizations between different ports of the same system. However, different systems shall communicate only through channels.

\subsection{Syntax and Operational Semantics}

We consider a set $A$ of channels denoted by $a, b 1$, and a set $R$ of resources denoted by $r, k$. The syntax of the calculus uses three syntactic categories: threads, processes and handshake configurations. Threads are purely sequential and allow prefixing while processes are parallel compositions of threads. The prefixes are input and output actions on a finite set of resources. As we will see later, input actions release resources and output actions use resources. Let $\Delta \subseteq A$ :

$$
\begin{aligned}
a c t: & :=a^{\left\{r_{1}, \ldots, r_{n}\right\}} \mid \bar{a}_{\left\{r_{1}, \ldots, r_{n}\right\}} \text { Actions } \\
T:: & =\mathbf{0} \mid \text { act.T } \mid \text { Rec } T \text { Threads } \\
P, Q & :=T|P| Q \mid P \backslash \Delta \text { Processes }
\end{aligned}
$$

A handshake configuration is composed of a process $P$ along with a multiset of resources $S$ for internal synchronization. A configuration can be open or closed:

$$
M::=\left\{P, S \int \mid\langle P, S\rangle \quad\right. \text { Open and closed configurations }
$$

\footnotetext{
${ }^{1}$ We will use channels to model ports, but we prefer to keep the conceptual difference between the two notions.
} 


$$
\begin{aligned}
& \overline{\left\{a^{\left\{r_{1}, \ldots, r_{n}\right\}} . T, S \int \stackrel{a}{\longrightarrow}\left\{T, S+\left\{r_{1}, \ldots, r_{n}\right\}\right\}\right.} \text { (inev) } \\
& \frac{2 T \cdot \operatorname{Rec} T, S \int \stackrel{e}{\longrightarrow}\left\{T^{\prime}, S^{\prime} S\right.}{2 \operatorname{Rec} T, S \int \stackrel{e}{\longrightarrow}\left\{T^{\prime}, S^{\prime} S\right.}(\mathrm{rec}) \\
& \overline{\left\{\bar{a}_{\left\{r_{1}, \ldots, r_{n}\right\}} \cdot T, S+\left\{r_{1}, \ldots, r_{n}\right\} S \stackrel{\bar{a}}{\longrightarrow}\{T, S S\right.} \text { (outev) } \\
& \frac{M \stackrel{e}{\longrightarrow} M^{\prime} \quad \operatorname{ch}(e) \notin \Delta}{M \backslash \Delta \stackrel{e}{\longrightarrow} M^{\prime} \backslash \Delta} \text { (res) } \\
& \frac{M \stackrel{e}{\longrightarrow} M^{\prime}}{M\left|N \stackrel{e}{\longrightarrow} M^{\prime}\right| N} \text { (par1) } \\
& \frac{P \equiv P^{\prime} \quad\left\{P^{\prime}, S \int \stackrel{e}{\longrightarrow} 2 Q^{\prime}, S^{\prime} S \quad Q \equiv Q^{\prime}\right.}{2 P, S S \stackrel{e}{\longrightarrow} 2 Q, S^{\prime} S} \text { (struct) } \\
& \frac{M \stackrel{\bar{a}}{\longrightarrow} M^{\prime} \quad N \stackrel{a}{\longrightarrow} N^{\prime}}{M\left|N \stackrel{\tau}{\longrightarrow} M^{\prime}\right| N^{\prime}}(\text { par2) } \\
& \frac{\left\langle P, S \int \stackrel{e}{\longrightarrow}\left\{Q, S^{\prime} S\right.\right.}{\langle P, S\rangle \stackrel{e}{\longrightarrow}\left\langle Q, S^{\prime}\right\rangle} \text { (closure) }
\end{aligned}
$$

Fig. 1. Labeled transition semantics

Intuitively, open configurations represent systems under construction, whose resources are still accessible to the environment. Closed configurations represent completed systems and can only communicate via handshake channels.

The operational semantics is given in terms of an LTS over handshake configurations. Labels are channels with their polarity, plus the unobservable label:

$$
e::=\bar{a}|a| \tau \quad a \in A
$$

Given an observable label, the function $c h$ returns the channel on which it occurred. Formally: $\operatorname{ch}(\bar{a})=\operatorname{ch}(a)=a$ for any channel $a$. The definition of the operational semantics is simplified thanks to the congruence ( $\equiv$ ) between processes:

$$
P|Q \equiv Q| P \quad \operatorname{Rec} \mathbf{0} \equiv \mathbf{0}
$$

Let $\operatorname{res}(P)$ be the set of resources of a process $P$. As meta-notation, we define sequential composition of threads $T \cdot T^{\prime}$ :

$($ act. $T) \cdot T^{\prime}=\operatorname{act} .\left(T \cdot T^{\prime}\right) \quad T \cdot T^{\prime}=T^{\prime}(T \equiv \mathbf{0}) \quad(\operatorname{Rec} T) \cdot T^{\prime}=\operatorname{Rec} T(T \not \equiv \mathbf{0})$

and we extend process operators to configurations:

$$
\begin{array}{ll}
\left\langle P_{1}, S_{1} S\right|\left\langle P_{2}, S_{2} S=\left\{P_{1} \mid P_{2}, S_{1}+S_{2} S\right.\right. & \left\{P, S \int \backslash \Delta=\{P \backslash \Delta, S S\right. \\
\left\langle P_{1}, S_{1}\right\rangle \mid\left\langle P_{2}, S_{2}\right\rangle=\left\langle\mu_{1}\left(P_{1}\right) \mid \mu_{2}\left(P_{2}\right), \mu_{1}\left(S_{1}\right)+\mu_{2}\left(S_{2}\right)\right\rangle & \langle P, S\rangle \backslash \Delta=\langle P \backslash \Delta, S\rangle
\end{array}
$$

where + denotes the union of multisets and $\mu_{1}: \operatorname{res}\left(P_{1}\right) \cup S_{1} \rightarrow R_{1}$ and $\mu_{2}: \operatorname{res}\left(P_{2}\right) \cup$ $S_{2} \rightarrow R_{2}$ are injective functions between resources such that $R_{1}$ and $R_{2}$ are disjoint and all the resources they contain are fresh. Moreover $\mu(S)$ is the point-to-point application of the function $\mu$ to the multiset $S$, while $\mu(P)$ is the process obtained from $P$ by renaming any label occurrence according to $\mu$. This guarantees that two closed configurations can only communicate via channels . 


\begin{tabular}{cc}
\hline$\frac{a \in A}{\mathbf{0} \triangleright ! a}$ (ax) & $\frac{T \triangleright ! a}{\operatorname{Rec} T \triangleright ! a}$ (rec) \\
$\frac{T \triangleright ? a}{\bar{a}_{\left\{r_{1}, \ldots r_{n}\right\}} \cdot T \triangleright ! a}$ (outpref) & $\frac{T \triangleright ! a}{a^{\left\{r_{1}, \ldots r_{n}\right\}} \cdot T \triangleright ? a}$ (inpref) \\
$\left.\frac{P \triangleright \Gamma^{\prime} \quad Q \triangleright \Gamma^{\prime \prime}}{(P \mid Q) \backslash\left(\operatorname{Dom}\left(\Gamma^{\prime}\right) \cap \operatorname{Dom}\left(\Gamma^{\prime \prime}\right)\right) \triangleright \Gamma^{\prime} \odot \Gamma^{\prime \prime}}\right)$ \\
$\frac{P \triangleright \Gamma \Gamma^{\prime \prime}(a)}{2 P, S \int \triangleright \Gamma}$ (oconf) \\
\hline \hline
\end{tabular}

Fig. 2. Handshake types

Note that we do not define the parallel composition of an open and a closed configuration. The idea is that we may combine two different parts of a system (in the construction stage) or two completed systems (for interaction), but we may not combine a system under construction with a completed one.

The derivation rules for the operational semantics are shown in Figure 1 . When an input occurs, a set of resources becomes available; while an output requires a set of resources in order to occur, then the used resources disappear. The other rules are quite standard. Note however that the operational distinction between open and closed configurations comes from the two distinct cases of composition given above. In the parallel composition of open configurations, one side may influence the other by modifying a shared resource, as no renaming takes place. This is not possible for closed configurations, as renaming prevents the sharing of resources.

A sequence of transitions $M_{0} \stackrel{e_{0}}{\longrightarrow} M_{1} \ldots M_{n} \stackrel{e_{n}}{\longrightarrow} M$ is denoted $M_{0} \stackrel{t}{\rightarrow} M$, where $t=$ $e_{0} \ldots e_{n}$. The string $t$ is called the strong trace of the sequence, while the weak trace is the restriction of $t$ to the labels other than $\tau$. Strong $(\sim)$ and weak $(\approx)$ bisimilarity are also defined as usual [13] on the labeled transition system for $\mathrm{CHC}$.

\subsection{Typing System}

A type $\Gamma$ is a partial function from channel names to $\{!, ?\}$. We will use the shorthand notation $! a$ or $? a$ to describe a type defined on channel $a$, and commas to join types. We say that $a$ is active in $\Gamma$ when $\Gamma(a)=$ ! and we say it is passive when $\Gamma(a)=$ ?.

Let $\Gamma^{\prime}$ and $\Gamma^{\prime \prime}$ be two types and let $a$ be a channel. Let us define the function $\Gamma^{\prime} \odot \Gamma^{\prime \prime}$ : $\left(\operatorname{Dom}\left(\Gamma^{\prime}\right) \backslash \operatorname{Dom}\left(\Gamma^{\prime \prime}\right)\right) \cup\left(\operatorname{Dom}\left(\Gamma^{\prime \prime}\right) \backslash \operatorname{Dom}\left(\Gamma^{\prime}\right)\right) \rightarrow\{$ !, ?\} such that:

- $\Gamma^{\prime} \odot \Gamma^{\prime \prime}(a)=\Gamma^{\prime}(a)$, when $a \in \operatorname{Dom}\left(\Gamma^{\prime}\right) \backslash \operatorname{Dom}\left(\Gamma^{\prime \prime}\right)$;

- $\Gamma^{\prime} \odot \Gamma^{\prime \prime}(a)=\Gamma^{\prime \prime}(a)$, when $a \in \operatorname{Dom}\left(\Gamma^{\prime \prime}\right) \backslash \operatorname{Dom}\left(\Gamma^{\prime}\right)$.

Typing judgements are of the form $T \triangleright \Gamma, P \triangleright \Gamma, M \triangleright \Gamma$, where $T$ is a thread, $P$ a process, $M$ a configuration and $\Gamma$ a type. The typing rules are shown in Figure 2 The empty thread is active: this models receptiveness, because a thread of passive type 
must always be able to perform another input. The following three rules guarantee that threads are alternating on each channel. The parallel composition of two processes is allowed only if threads on the same channel have dual types. These channels must then be restricted so that no other process can communicate on them. This models the pointto-point communication discipline of handshake protocols. Note that resources do not play any role in the typing.

The following results show the intuition behind the typing system.

Lemma 3 (Reduction). Let $M$ be a configuration such that $M \triangleright \Gamma$. Then:

$$
\begin{array}{ll}
-M \stackrel{a}{\longrightarrow} \Longleftrightarrow \Gamma(a)=? ; & \\
-M \stackrel{a}{\longrightarrow} \Rightarrow \Gamma(a)=! ; & \\
-M \stackrel{e}{\longrightarrow} M^{\prime} \wedge e \neq \tau \Rightarrow M^{\prime} \triangleright \Gamma^{\prime} \text { s.t. } & \operatorname{Dom}\left(\Gamma^{\prime}\right)=\operatorname{Dom}(\Gamma) \wedge \\
& \forall b \in \operatorname{Dom}(\Gamma), b \neq \operatorname{ch}(e) \leftrightarrow \Gamma(b)=\Gamma^{\prime}(b) ; \\
-M \stackrel{\tau}{\longrightarrow} M^{\prime} \Rightarrow M^{\prime} \triangleright \Gamma ; &
\end{array}
$$

Corollary 4 (Subject Reduction). Let $M \triangleright \Gamma$ and $M \stackrel{s}{\longrightarrow} M^{\prime}$ then there is a type $\Gamma^{\prime}$ such that $M^{\prime} \triangleright \Gamma^{\prime}$.

\subsection{Examples}

As a first example, we show a configuration representing the $O R$ handshake protocol.

$$
O R=\left\langle a^{\left\{r_{1}\right\}} \cdot \operatorname{Rec} \bar{a}_{\left\{r_{2}\right\}} \cdot a^{\left\{r_{1}\right\}} \cdot \mathbf{0}\left|\operatorname{Rec} \bar{b}_{\left\{r_{1}\right\}} \cdot b^{\left\{r_{2}\right\}} \cdot \mathbf{0}\right| \operatorname{Rec} \bar{c}_{\left\{r_{1}\right\}} \cdot c^{\left\{r_{2}\right\}} \cdot \mathbf{0}, \emptyset\right\rangle
$$

We have that $O R \triangleright ? a, ! b, ! c$. When a request on the passive port $a$ arrives, the resource $r_{1}$ becomes available and this enables $O R$ to send a request on either active port $b, c$. An acknowledge to this last request enables an acknowledge to the first one. The second configuration represents the $M I X$ protocol.

$$
M I X=\left\langle b^{\left\{k_{1}\right\}} \cdot \operatorname{Rec} \bar{b}_{\left\{k_{2}\right\}} \cdot b^{\left\{k_{1}\right\}} \cdot \mathbf{0}\left|c^{\left\{k_{1}\right\}} \cdot \operatorname{Rec} \bar{c}_{\left\{k_{2}\right\}} \cdot c^{\left\{k_{1}\right\}} \cdot \mathbf{0}\right| \operatorname{Rec} \bar{d}_{\left\{k_{1}\right\}} \cdot d^{\left\{k_{2}\right\}} \cdot \mathbf{0}, \emptyset\right\rangle
$$

We have that $M I X \triangleright ? b, ? c, ! d$. Each time an environment request arrives (on either passive port $b, c)$, the component $M I X$ handshakes on its active port $d$ and after completion it acknowledges to the first request. If, by the time the handshake on the active port is complete, the environment has sent a request on the other port, $M I X$ chooses nondeterministically which request to acknowledge first.

The two protocols can be composed in parallel, communicating on the common ports. We have $(O R \mid M I X) \backslash\{b, c\} \triangleright ? a, ! d$.

\subsection{Soundness}

In this section we show that typed CHC configurations indeed define handshake languages, using the weak traces of the labeled transition semantics.

Each feature of the calculus plays a role in modeling the handshake discipline. Let us see informally how. First of all, handshake languages alternate input and output on the same port. This is enforced by the typing system. The reorder closure is guaranteed by 
the fact that different ports are on different parallel threads. The only reordering that is in general not allowed is when an input blocks an output. An input can block an output because an output may need resources that will only be provided by the input. Finally, receptiveness is guaranteed by the fact that inputs do not need resources, and can always occur, provided that the alternation with the corresponding outputs is respected.

In order to denote handshake languages we consider the weak traces of the transition sequences of a configuration. If we considered the traces of all transition sequences, the denoted languages would always be prefix closed and some handshake languages would excape us. To characterize the larger class of languages closed under passive prefixes, we consider only the traces of the quiescent transition sequences.

A configuration $M$ is quiescent if it cannot (weakly) perform an output, i.e. if there is no transition sequence of the form $M \stackrel{(\tau)^{*}}{\longrightarrow} \stackrel{\bar{a}}{\longrightarrow}$, for any channel $a$. A transition sequence $M \stackrel{t}{\longrightarrow} M^{\prime}$ is quiescent if $M^{\prime}$ is.

Definition 5. Let $M$ be a handshake configuration. We define $H L(M)$ to be the set of weak traces of all the quiescent transition sequences which start from $M$.

Let $M$ be a configuration and $\Gamma$ a type such that $M \triangleright \Gamma$. The handshake structure $H S(\Gamma)=\left\langle\operatorname{Port}_{\Gamma}, d_{\Gamma}\right\rangle$ is defined by setting $\operatorname{Ports}_{\Gamma}=\operatorname{Dom}(\Gamma)$ and $d_{\Gamma}=\Gamma$.

Proposition 6 (Soundness). Let $M$ be a handshake configuration, such that $M \triangleright \Gamma$. Then $H L(M)$ is a handshake language on the handshake structure $H S(\Gamma)$.

We observe, however, that the other direction, the fact that each language is the denotation of some configuration, cannot be established. This is due to the presence of non recursive handshake languages which could never be captured by finite configurations. It would still be interesting to characterize the class of handshake languages that correspond to $\mathrm{CHC}$ configurations. We leave this as future work.

\subsection{Compositionality}

Open configurations can communicate via shared resources, but this is not directly observable in the labeled transition semantics. Thus we cannot expect a labeled equivalence to be fully congruent for them. However weak bisimilarity is a congruence with respect to composition of closed configurations:

Proposition 7. Let $M_{1}, M_{2}, N$ be closed handshake configurations such that $M_{1}, M_{2} \triangleright$ $\Gamma, N \triangleright \Gamma^{\prime}$ and $\left(M_{1} \mid N\right) \backslash \Delta \triangleright \Gamma \odot \Gamma^{\prime},\left(M_{2} \mid N\right) \backslash \Delta \triangleright \Gamma \odot \Gamma^{\prime}$, where $\Delta=\operatorname{Dom}(\Gamma) \cap \operatorname{Dom}\left(\Gamma^{\prime}\right)$. Then $M_{1} \approx M_{2}$ implies $\left(M_{1} \mid N\right) \backslash \Delta \approx\left(M_{2} \mid N\right) \backslash \Delta$.

This is consistent with our intepretation of resources as internal means of communication. Our main goal was to describe the externally observable behavior of a system and we do so by considering only those configurations whose resources cannot be accessed by the environment.

In Section 2 we talked about the difficulty of finding a good definition of composition for handshake languages. This intuition is confirmed as "quiescent trace equivalence" is not a congruence. Consider the following processes:

$$
\begin{gathered}
P_{1}=\bar{c}_{\left\{r_{1}, r_{2}\right\}} \cdot c^{\{\}} \cdot \mathbf{0}\left|\bar{b}_{\left\{r_{3}, r_{1}\right\}} \cdot b^{\left\{r_{1}\right\}} \cdot \operatorname{Rec} \bar{b}_{\left\{r_{1}\right\}} \cdot b^{\left\{r_{1}\right\}} \cdot \mathbf{0}\right|\left(\bar{d}_{\left\{r_{3}, r_{2}\right\}} \cdot d^{\left\{r_{3}\right\}} \cdot \mathbf{0} \mid d^{\{\}} \cdot \bar{d}_{\{\}} \cdot d^{\{\}} \cdot \mathbf{0}\right) \backslash\{d\} \\
P_{2}=\bar{c}_{\left\{r_{1}\right\}} \cdot c^{\{\}} \cdot \mathbf{0} \mid \operatorname{Rec} \bar{b}_{\left\{r_{1}\right\}} \cdot b^{\left\{r_{1}\right\}} \cdot \mathbf{0} .
\end{gathered}
$$


Consider the closed configurations $M_{1}=\left\langle P_{1},\left\{r_{1}, r_{2}, r_{3}\right\}\right\rangle$ and $M_{2}=\left\langle P_{2},\left\{r_{1}, r_{2}, r_{3}\right\}\right\rangle$. They are both interpreted as the same handshake language:

$$
H L\left(M_{1}\right)=\{\bar{c}, \bar{c} c, \bar{b}, \bar{b} b \bar{c}, \bar{b} b \bar{c} c, \bar{b} b \bar{b}, \ldots\}=H L\left(M_{2}\right)
$$

however, if we compose them with $N=\left\langle b^{\{\}} \cdot \operatorname{Rec} \bar{b}_{\{\}} \cdot b^{\{\}} . \mathbf{0}\left|c^{\{k\}} . \mathbf{0}\right| \bar{a}_{\{k\}} \cdot a^{\{\}} . \mathbf{0}, \emptyset\right\rangle$ we obtain two configurations with different interpretations:

$$
H L\left(\left(M_{1} \mid N\right) \backslash\{b, c\}\right)=\{\varepsilon, \bar{a}, \bar{a} a\} \quad H L\left(\left(M_{2} \mid N\right) \backslash\{b, c\}\right)=\{\bar{a}, \bar{a} a\}
$$

Therefore the parallel composition of CHC configurations cannot be used to define the composition of handshake languages. In order to compose handshake protocols, some more knowledge on the branching structure is needed. CHC provides a suitable formalism to study this structure.

\section{Handshake Petri Nets}

We argued that not all handshake languages can be represented by $\mathrm{CHC}$ configurations, as, for instance, there are non recursive handshake languages. To show the expressive power of our calculus, we provide an alternative semantics of $\mathrm{CHC}$ based on Petri nets. In [6], we studied a Petri net representation of handshake protocols, called handshake Petri net, and we showed that all handshake languages can have a, possibly infinite, handshake Petri net representation. In this paper we show that every finite handshake Petri net is weakly bisimilar to a CHC configuration.

In this section we introduce handshake Petri nets. The present definition is slightly different from the one in [6], but the results of that paper carry over. We assume some basic knowledge on Petri nets, which we will use in their standard graphical representation [15]. Throughout the paper we will consider Petri nets in their unsafe version, where places are allowed to contain several tokens at the same time. This is not just for convenience. Unsafe nets are necessary to carry out our construction.

\subsection{Definition}

Handshake Petri nets are Petri nets with a special "external interface", reflecting the structure of handshake ports. We define handshake ports in two phases. We first define the static structure of ports, and then we specify the markings.

Let $G$ be a Petri net and let $N_{O}$ and $N_{I}$ be a partition of its nodes (places and transitions). The elements of $N_{O}$ will be called output transitions / places while the elements of $N_{I}$ will be called input transitions / places. We give an inductive definition of a static handshake port $a=\left\langle G, N_{O}, N_{I}\right\rangle$ as follows:

- (Basic cases) $N_{O}$ and $N_{I}$ contain no transition;

- (Inductive cases) let $a^{\prime}=\left\langle G^{\prime}, N_{O}^{\prime}, N_{I}^{\prime}\right\rangle$ be a static port:

- (input prefixing) given a place $p \in N_{I}^{\prime}$ with no outgoing arcs, $a$ is obtained from $a^{\prime}$ by adding an input transition $t$ and an arc from $p$ to $t$; 


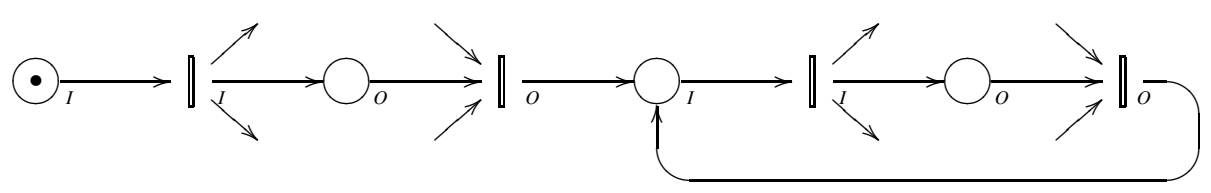

Fig. 3. A passive port ( $I$ is for input and $O$ is for output)

- (output prefixing) given a place $p \in N_{O}^{\prime}$ with no outgoing arcs and a place $p^{\prime} \in N_{I}^{\prime}$ with exactly one outgoing arc, $a$ is obtained from $a^{\prime}$ by adding an output transition $t$, an arc from $p$ to $t$ and an arc from $t$ to $p^{\prime}$;

- (alternation) given a place $p \in N_{O}^{\prime}$ and a transition $t \in N_{I}^{\prime}$ with no outgoing arcs, $a$ is obtained from $a^{\prime}$ by adding an arc from $t$ to $p$.

Let $a^{\prime}$ be a static port and let $p$ be a place of (the Petri net of) $a^{\prime}$ such that if $p$ is an input place, $p$ has an outgoing arc. Let $a$ be the net obtained from $a^{\prime}$ either by adding one token to $p$ or by keeping $a^{\prime}$ with no tokens, then $a$ is a handshake port. Moreover, if $a$ is as $a^{\prime}$ (no tokens) or if $p$ is an output place we say that $a$ is an active port, otherwise we say that $a$ is a passive port.

Let $G$ be a Petri net, $G^{\prime}$ a subgraph of $G$ and $a=\left\langle G^{\prime}, N_{O}, N_{I}\right\rangle$ a port s.t. :

- a place of $G^{\prime}$ may only be connected to transitions of $G^{\prime}$;

- a transition $t \in N_{O}$ of $G^{\prime}$ may only have outgoing arcs to places of $G^{\prime}$;

- a transition $t \in N_{I}$ of $G^{\prime}$ may only have incoming arcs from places of $G^{\prime}$.

then $a$ is a handshake port of $G$. Figure 3 shows an example of a passive handshake port of some net. The arrows without source or target indicate the way the port may connect to the rest of the net. The statical structure imposes alternation between the firings of input and output transitions. It is also ensured that, if an input place may ever contain a token, then it must have an outgoing arc to an input transition (receptiveness). Finally, by allowing a port to contain several input and output transitions we are able to model each event separately. For instance, two distinct input transitions may connect differently to the rest of the net, thus providing different resources.

Definition 8. The pair $H=\left\langle G_{H}\right.$, Port $\left.s_{H}\right\rangle$ is a handshake Petri net (hpn) just when $G_{H}$ is a Petri net and Ports ${ }_{H}$ a set of disjoint handshake ports of $G_{H}$.

Let $H=\left\langle G_{H}\right.$, Ports $\left.s_{H}\right\rangle$ and let $t(p)$ be a transition (place) of $G_{H}$. Then $t(p)$ is internal of $H$ if it is neither of input nor of output (in some port $a$ of $H$ ).

\subsection{Composition}

A linkage between an active port $a \in$ Ports $_{H}$ and a passive port $b \in$ Port $_{H}$ of an hpn $H=\left\langle G_{H}\right.$, Ports $\left._{H}\right\rangle$ is the hpn $L(H, a, b)=\left\langle G_{H, a, b}\right.$, Port $\left._{H} \backslash\{a, b\}\right\rangle$, where $G_{H, a, b}$ is the net obtained by adding two fresh places $p_{1}$ and $p_{2}$ to $G_{H}$ and arcs from each output transition of $a$ to $p_{1}$, from $p_{1}$ to each input transition of $b$, from each output transition of $b$ to $p_{2}$ and from $p_{2}$ to each input transition of $a$.

We call link of $L(H, a, b)$, denoted $\operatorname{link}(H, a, b)$, the graph consisting of the graphs of $a$ and $b$ plus $p_{1}, p_{2}$ and any arc connecting them to transitions of $a$ or $b$. Figure 4 shows an example of link between an active port (left) and a passive port (right). 


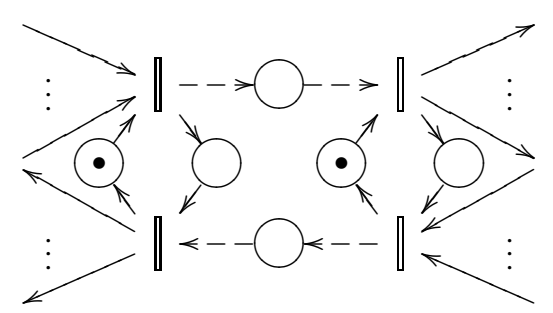

Fig. 4. Example of composition of ports

Definition 9. Let $H_{1}=\left\langle G_{1}\right.$, Port $\left.s_{1}\right\rangle$ and $H_{2}=\left\langle G_{2}\right.$, Port $\left.s_{2}\right\rangle$ be two handshake Petri nets. Let $\left\{a_{1}, \ldots a_{n}\right\} \subseteq$ Ports $_{1} \cup$ Ports $_{2}$ be a set of active handshake ports and let $\left\{b_{1}, \ldots b_{n}\right\} \subseteq$ Port $_{1} \cup$ Port $_{2}$ be a set of passive handshake ports, such that for $1 \leq$ $i \leq n, a_{i} \in$ Ports $_{1}$ if and only if $b_{i} \in$ Port $_{2}$. Then:

$$
\begin{gathered}
H_{1} \|_{\left\{\left(a_{1}, b_{1}\right), \ldots\left(a_{n}, b_{n}\right)\right\}} H_{2} \\
= \\
L\left(\ldots L\left(\left\langle G_{1}+G_{2}, \text { Ports }_{1} \cup \text { Ports }_{2}\right\rangle, a_{1}, b_{1}\right), \ldots a_{n}, b_{n}\right)
\end{gathered}
$$

is the composition of $H_{1}$ with $H_{2}$ by linking the pairs $\left(a_{1}, b_{1}\right), \ldots\left(a_{n}, b_{n}\right)$.

It is easy to see that the composition of two hpns is well-defined and associative.

\subsection{Observational Properties of hpns}

Let us label $a(\bar{a})$ each input (output) transition of port $a$, for any $a \in \operatorname{Ports}_{H}$, and $\tau$ each internal transition of $H$. We write $H \stackrel{l}{\rightarrow} H^{\prime}$ (which reads $H l$-reduces to $H^{\prime}$ ) if a transition labeled $l$ is enabled in $G_{H}$ and its firing leads to the hpn $H^{\prime}$. Seen as labeled transition systems, hpns naturally inherit many definitions that we gave for CHC. Two of these are strong $(\sim)$ and weak $(\approx)$ bisimilarity. The generality of these definitions allow us to go as far as saying that an hpn is (weakly or strongly) bisimilar to a handshake configuration.

Another inherited definition is that of the function $H L$, which is identical to the one we gave in Section 3.4 for a handshake configuration. However, we still need to adapt $H S$. Let $H=\left\langle G_{H}\right.$, Port $\left._{H}\right\rangle$ be an hpn and let $d_{H}:$ Port $_{H} \rightarrow\{!, ?\}$ be the function which maps each port $a$ to !, when $a$ is active in $H$, or to ?, when it is passive. We define $H S(H)=\left\langle\right.$ Port $\left._{H}, d_{H}\right\rangle$.

Proposition 10. [6] Let $H$ be a handshake Petri net, then $H L(H)$ is a handshake language on the handshake structure $H S(H)$.

Proposition 11. [6] Let $\sigma$ be a handshake language on a handshake structure $\langle P, d\rangle$. Then there is an hpn $H_{\sigma}$ such that $H S\left(H_{\sigma}\right)=\langle P, d\rangle$ and $H L\left(H_{\sigma}\right)=\sigma$.

Note that, for the last result, the net $H_{\sigma}$ may contain an infinite number of internal places and transitions. This is unavoidable as languages are in general not recursive and thus not finitely representable. Figure 5 shows two simple examples of handshake protocols: $O R$ and MIX described in Section 3.3 

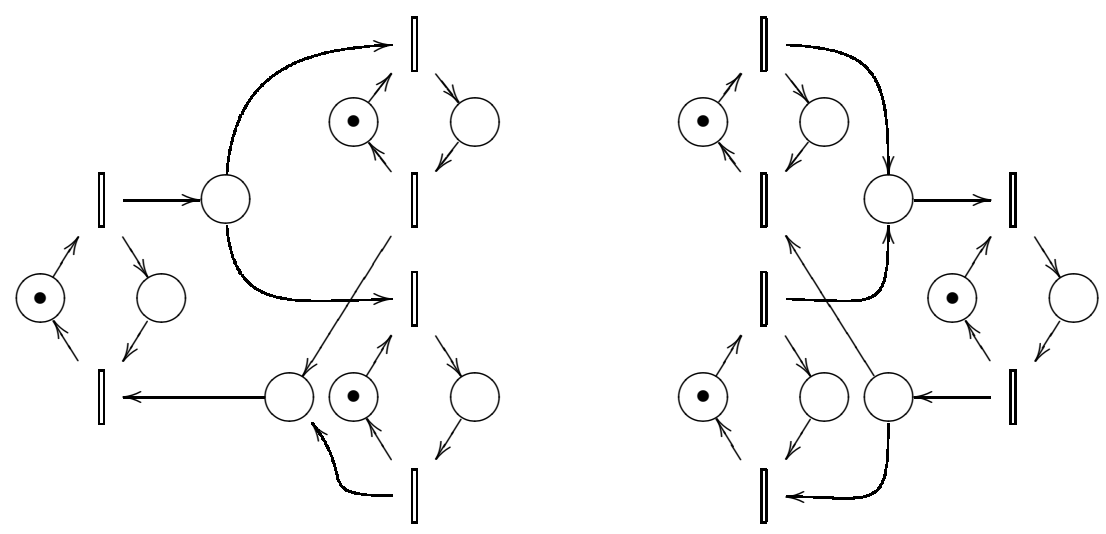

Fig. 5. OR (left) and $M I X$ (right) handshake components

\section{Full Abstraction and Definability}

In this section we relate the calculus $\mathrm{CHC}$ with its Petri nets model. We only sketch the constructions, the detailed proofs are available on the extended version [5].

\subsection{Full Abstraction}

Let $M$ be a handshake configuration, such that $M \triangleright \Gamma$. We can assume $M=\langle P, S\rangle$ and define the hpn $\llbracket M \rrbracket_{\Gamma}$ by cases of $P$. The definitions for open configurations are identical, $\llbracket 2 P, S \int \rrbracket_{\Gamma}=\llbracket\langle P, S\rangle \rrbracket_{\Gamma}$.

Let $P=\mathbf{0}$. Then $\Gamma=! a$ for some channel $a$. Now, define a port which contains a single output place $p$ holding a token and call it $a$ as the channel. Let $G$ be the Petri net which contains $p$ plus an internal place $q$, labeled $r$, for each $r \in S$, where $q$ contains as many tokens as there are occurrences of $r$ in $S$. Then $\llbracket M \rrbracket_{\Gamma}$ is the hpn $\langle G,\{a\}\rangle$.

Let $P=a^{\left\{r_{1}, \ldots r_{n}\right\}} \cdot P^{\prime}$. Then the last applied typing rule is (inpref), then $\Gamma=? a$ and $P^{\prime} \triangleright ! a$. Let $\llbracket\left\langle P^{\prime}, S\right\rangle \rrbracket_{! a}=\left\langle G^{\prime}\right.$, Port $\left.s^{\prime}\right\rangle$. By construction, Ports $s^{\prime}=\{a\}$ where $a$ is an active port. Let $p^{\prime}$ be the place of $a$ with a token. Let's extend $a$ by adding a fresh input place $p$, a fresh input transition $t$ labeled $a$ and arcs from $p$ to $t$ and from $t$ to $p^{\prime}$, then by removing the token from $p^{\prime}$ and putting a token into $p$. Finally let's add arcs from $t$ to any place labeled $r_{i}$, for $1 \leq i \leq n$. If any of these places does not exist yet, add it anew. We thus obtain a graph $G$. Then $\llbracket M \rrbracket_{\Gamma}=\langle G,\{a\}\rangle$. The case of the output prefix is dual.

Let $P=\operatorname{Rec} P^{\prime}$. Then $\Gamma=! a$ and $P^{\prime} \triangleright ! a$, for some channel $a$. Let $\llbracket\left\langle P^{\prime}, S\right\rangle \rrbracket ! a=$ $\left\langle G^{\prime}\right.$, Ports $\left.^{\prime}\right\rangle$. By construction, Ports ${ }^{\prime}=\{a\}$ where $a$ is also the active port associated to channel $a$. Let $p$ be the place of $a$ which holds a token. If $p$ has an incoming arc or if any other place in $a$ has two incoming arcs, $\llbracket\langle P, S\rangle \rrbracket_{\Gamma}=\llbracket\left[P^{\prime}, S\right\rangle \rrbracket_{! a}$. Otherwise $a$ must contain a place $p^{\prime}$ with no outgoing arcs, by construction. Note that both $p$ and $p^{\prime}$ must be output places, also by construction. Then replace $p$ and $p^{\prime}$ by a place $q$ obtained by “joining" them. In particular, $q$ must be the new source of $p$ 's outgoing arc and the new target of $p^{\prime}$ 's incoming arc. Call $G$ the graph so obtained. Then $\llbracket\langle P, S\rangle \rrbracket_{\Gamma}=\langle G,\{a\}\rangle$. 
Let $P=\left(P^{\prime} \mid P^{\prime \prime}\right) \backslash \Delta$. We construct $\llbracket\langle P, S\rangle \rrbracket_{\Gamma}$ in three steps. First let $\llbracket\left\langle P^{\prime}, S\right\rangle \mathbb{1}_{\Gamma_{1}}^{\Delta}$ be obtained from $\llbracket\left\langle P^{\prime}, S\right\rangle \rrbracket_{\Gamma_{1}}$ by renaming each port $a$ such that $(a, a) \in \Delta$, as $a^{\text {! }}$ when $\Gamma_{1}(a)=$ ! and as $a^{\text {? }}$ when $\Gamma_{1}(a)=$ ?. Define analogously $\mathbb{L}\left\langle P^{\prime \prime}, S\right\rangle \mathbb{1}_{\Gamma_{2}}^{\Delta}$. Then let

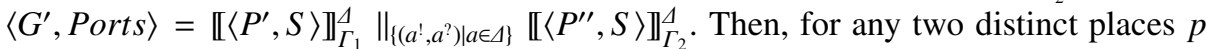
and $p^{\prime}$ of $G^{\prime}$ labeled by the same resource $r$ do the following: substitute $p$ and $p^{\prime}$ by a single place also labeled by $r$, having all the arcs of both $p$ and $p^{\prime}$; note also that by construction, $p$ and $p^{\prime}$ contained the same number of tokens $k$, then put $k$ tokens in the new place as well. Let $G$ be the net so obtained. Then $\llbracket M \rrbracket_{\Gamma}=\langle G$, Ports $\rangle$.

The semantics is well defined and fully abstract with respect to weak bisimilarity:

Lemma 12. Let $M$ be a configuration such that $M \triangleright \Gamma$. Then $\llbracket M \rrbracket_{\Gamma}$ as defined above is a handshake Petri net and $M \approx \llbracket M \rrbracket_{\Gamma}$.

Theorem 13 (Full Abstraction). Let $M$ and $M^{\prime}$ be two configurations such that $M \triangleright \Gamma$ and $M^{\prime} \triangleright \Gamma^{\prime}$. Then $M \approx M^{\prime} \Longleftrightarrow \llbracket M \rrbracket_{\Gamma} \approx \llbracket M^{\prime} \rrbracket_{\Gamma^{\prime}}$.

\subsection{Definability}

For each finite hpn there is a weakly bisimilar handshake configuration:

Theorem 14 (Definability). Let $H=\langle G$, Ports $\rangle$ be a handshake Petri net. Then there are a closed handshake configuration $M$ and a handshake type $\Gamma$, such that $M \triangleright \Gamma$ and $\llbracket M \rrbracket_{\Gamma} \approx H$.

We present here a simplified construction of the configuration associated to $H$. The idea is that each port $a$ of the net $H$ can be modeled by a thread $\operatorname{Proc}(a, H)$, inductively on the structure of the port.

Each internal transition $t$ is first unfolded as a link between two ports and then associated to a process. Let $t$ have incoming arcs from internal places labeled $r_{1}, \ldots r_{i}$ and outgoing arcs to internal places labeled $r_{i+1}, \ldots r_{n}$. Then $t$ is unfolded as follows:
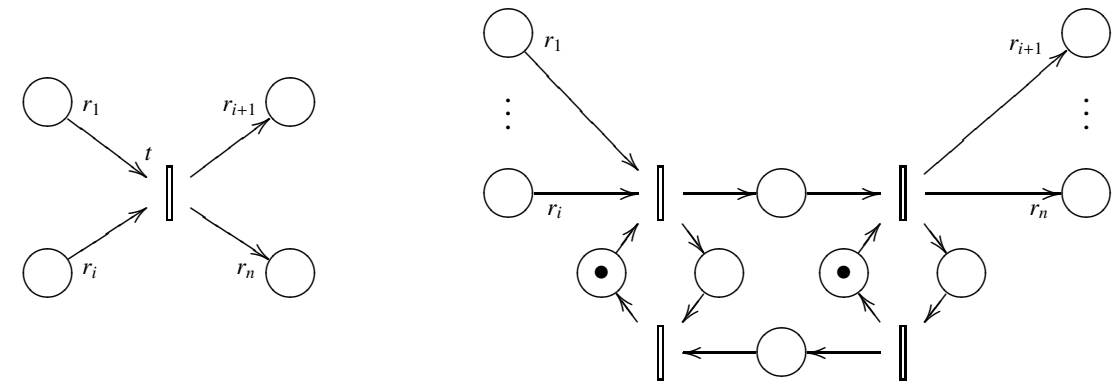

where $r_{1}, \ldots r_{n}$ are place labels. . Then let $H$ be a hpn, $u(H)$ is the hpn obtained from $H$ by unfolding each of its internal transitions. It can be shown that $H \approx u(H)$.

For each internal transition $t$, let $l_{t}$ be a fresh label associated to it. Consider the following process.

$$
\operatorname{Proc}(t, H)=\left(\boldsymbol{\operatorname { R e c }} \bar{l}_{t\left\{r_{1}, \ldots r_{i}\right\}} \cdot l_{t} \cdot \mathbf{0} \mid l_{t}^{\left\{r_{i+1}, \ldots r_{n}\right\}} \cdot \operatorname{Rec} \bar{l}_{t} \cdot l_{t}^{\left\{r_{i+1}, \ldots r_{n}\right\}} \cdot \mathbf{0}\right) \backslash\left\{l_{t}\right\}
$$


Then we define

$$
\operatorname{Proc}(H)=\operatorname{Proc}\left(a_{1}, H\right)|\ldots| \operatorname{Proc}\left(a_{n}, H\right)\left|\operatorname{Proc}\left(t_{1}, H\right)\right| \ldots \mid \operatorname{Proc}\left(t_{m}, H\right)
$$

where $a_{1}, \ldots a_{n}$ are the ports of $H$ and $t_{1}, \ldots t_{m}$ are the internal transitions of $H$. Then let $\operatorname{Conf}(H)=\left\langle\operatorname{Proc}(H), S_{H}\right\rangle$, where $S_{H}$ is the multiset of labels of internal places of $H$ with a token and a label appears in $S_{H}$ as many times as the number of tokens in the corresponding place. Finally let ch(Ports) be the set of names of ports in Ports, then $\Gamma_{H}: \operatorname{ch}($ Ports $) \rightarrow\{!, ?\}$ is the function which associates ! to its active ports' names and ? to its passive ports' names. It can be shown that $\llbracket \operatorname{Con} f(H) \rrbracket_{\Gamma_{H}} \approx u(H)$. Thus $\llbracket \operatorname{Conf}(H) \rrbracket_{\Gamma_{H}} \approx H$.

\section{Conclusions}

We presented the calculus $\mathrm{CHC}$ which describes handshake protocols of communication. We have given it an lts semantics and a Petri nets semantics in terms of handshake Petri nets. We have shown that every finite handshake Petri net corresponds to a closed configuration of the calculus.

We have argued that a branching semantics is necessary to understand handshake protocols, as the trace model cannot properly define composition. The calculus and the two semantics provide the necessary framework to formally study handshake protocols.

Our original aim had been to devise a typing system for CCS, that would ensure the handshake discipline. After many attempts, we came to believe that such typing system would be cumbersome, if at all possible. Consider in particular the MIX component defined in Section 4.3 It is essentially characterized by a form of inclusively disjunctive causality: The request sent on the active port causally depends on either of the requests received on the passive ports. However, if both are received, it cannot be established which of the two is actually the cause. This is in contrast with the fact that CCS can be modeled using safe occurrence nets (which correspond to stable event structures), where this kind of causality cannot be represented. Therefore we decided to use a second, different form of communication, in the form of shared resources.

As usual, a result opens new directions to inspect. We would like to characterize the handshake languages that are described by $\mathrm{CHC}$ configurations (and thus by finite nets). We would also like to study restrictions on the language or the types to characterize behavioral classes of protocols: deterministic, positional, free choice, etc. It would be interesting to extend the calculus with mobility primitives, like the ones of the $\pi$-calculus, and study its expressive power. We also would like to use the calculus to specify and prove the correctness of specific protocols.

Acknowledgments. We thank Roberto Amadio, Catuscia Palamidessi and Frank Valencia for their useful comments. We thank an anonymous referee for spotting a mistake in a previous version of our proofs. The first author would like to thank Simona Ronchi della Rocca, Felice Cardone, Pierre-Louis Curien for their valuable inputs and support. The second author acknowledges the support of the ANR project ParSec: ANR-06SETI-010-02. 


\section{References}

1. Abramsky, S., Gay, S., Nagarajan, R.: Interaction categories and the foundations of types concurrent programming. In: Proc. of the 1994 Marktoberdorf Summer School on Deductive Program Design, pp. 35-113. Springer, Heidelberg (1996)

2. Arun-Kumar, S., Hennessy, M.: An efficiency preorder for processes. Acta Informatica 29(9), 737-760 (1992)

3. Bardsley, A.: Balsa: an asynchronous circuit synthesis system. Master's thesis. Department of Computer Science, University of Manchester (1998)

4. Fossati, L.: Modeling the Handshake Protocol for Asynchrony. PhD thesis, Dip. di Informatica, Univ. di Torino \& Lab. Preuves Programmes et Systèmes (PPS), Univ. Paris 7 (2009)

5. Fossati, L., Varacca, D.: The calculus of handshake configurations (extended version) (2008), http://www.di.unito.it/ fossati/

6. Fossati, L., Varacca, D.: A Petri net model of handshake circuits. In: Proc. of First International Workshop on Interactive Concurrency Experience, ICE 2008. ENTCS. Elsevier, Amsterdam (to be published, 2008), http://www.di . unito.it/ fossati/

7. Gelernter, D.: Generative communication in Linda. ACM Transactions on Programming Languages and Systems 7(1), 80-112 (1985)

8. Ghica, D.R.: Geometry of synthesis: a structured approach to VLSI design. In: Proc. of POPL 2007, pp. 363-375. ACM Press, New York (2007)

9. http://www.handshakesolutions.com/

10. Josephs, M., Udding, J., Yantchev, Y.: Handshake algebra. Technical Report SBU-CISM93-1, School of Computing, Information Systems and Mathematics, South Bank University, London (1993)

11. Kobayashi, N., Pierce, B., Turner, D.: Linearity and the $\pi$-calculus. ACM Transactions on Programming Languages and Systems 21(5), 914-947 (1999)

12. Mackie, I.: The geometry of interaction machine. In: Proc. of POPL 1995, pp. 198-208. ACM Press, New York (1995)

13. Milner, R.: Communication and Concurrency, 2nd edn. Prentice-Hall, Englewood Cliffs (1991)

14. Milner, R., Sangiorgi, D.: Techniques for "weak bisimulation up-to". Revised version of a paper that appeared in: Cleaveland, W.R. (ed.) CONCUR 1992. LNCS, vol. 630. Springer, Heidelberg (1992); available on Sangiorgi's webpage

15. Reisig, W.: Petri Nets: An Introduction. Monographs in Theoretical Computer Science. An EATCS Series, vol. 4. Springer, Heidelberg (1985)

16. Roscoe, A.W.: Unbounded nondeterminism in CSP. Journal of Logic and Computation 3(2), 131-172 (1993); Previously appeared in Two Papers on CSP, tech. monograph PRG-67, Oxford University Computing Laboratory(July 1988)

17. Udding, J.: Classification and Composition of Delay-Insensitive Circuits. PhD thesis, Department of Math. and C.S., Eindhoven University of Technology, Eindhoven (1984)

18. Van Berkel, K.: Handshake Circuits: an Asynchronous Architecture for VLSI Design. Cambridge International Series on Parallel Computation, vol. 5. Cambridge University Press, Cambridge (1993)

19. Yoshida, N., Honda, K., Berger, M.: Linearity and bisimulation. In: Nielsen, M., Engberg, U. (eds.) FOSSACS 2002. LNCS, vol. 2303, pp. 417-433. Springer, Heidelberg (2002) 